\title{
COMPARAÇÃO DE ADESIVOS ESTRUTURAIS NA JUNÇÃO DE CHAPAS METÁLICAS
}

\author{
T. F. BEZERRA ${ }^{1}$, J. J. FIORI ${ }^{1}$ \\ ${ }^{1}$ Centro Universitário Padre Anchieta, Jundiaí, SP \\ E-mail para contato: juliano.fiori@anchieta.br
}

\begin{abstract}
RESUMO - Os adesivos estruturais permitem a união de diversos tipos de materiais, sendo uma solução para junção sem o uso de rebites, parafusos ou soldaduras. Neste trabalho foram avaliadas propriedades de adesivos de base acrílica (A), epóxi (E) e uretânica (U) na adesão de chapas metálicas, definindo-se qual obtém melhor compatibilidade e desempenho com o substrato. Foram avaliadas resistências ao cisalhamento $(\mathrm{C})$ e tração axial $(\mathrm{T})$ de juntas expostas à névoa salina e ambiente natural. $O$ teste de névoa salina foi realizado em equipamento Salt Spray, com exposição de 500 horas das amostras suspensas entre 15 e $30^{\circ}$ em relação à vertical e paralelas à direção principal do fluxo. As tensões médias $\left(\mathrm{kgf} / \mathrm{in}^{2}\right)$ obtidas foram de 1055 (A), 566 (E) e 976 (U) para C e de $29(\mathrm{~A}), 15(\mathrm{E})$ e $30(\mathrm{U})$ para $\mathrm{T}$ em ambiente natural. A exposição à névoa salina diminuiu a resistência em todas as amostras. Com relação ao tipo de falhas, A apresentou falha coesiva (FC) em C e falha de substrato (FS) em T; E teve falha adesiva (FA) em C e T; e U apresentou FA/FC em C e FS em T. Conclui-se que A teve melhor desempenho, sua força de tração foi superior ou semelhante a $\mathrm{U}$, e as falhas foram consideradas ideais.
\end{abstract}

\section{INTRODUÇÃO}

Adesivo é definido pela norma ASTM D907 (2012) como "uma substância capaz de unir materiais através da fixação de suas superfícies". Desse modo, a junção de materiais com adesivo é um processo de união em que materiais adesivos são colocados entre duas superfícies sobrepostas, que são aderidas pela ação do mesmo (Ebnesajjad, 2011; Skeist, 1989).

Com o avanço da tecnologia, surgiram os adesivos estruturais, uma nova alternativa para união de diversos tipos de materiais. A cada ano essa tecnologia esta mais introduzida no mundo, permitindo solucionar necessidades de adesão sem o uso de rebites, parafusos e soldaduras (Fruet, 2011; Cognard, 2006).

Um adesivo estrutural resiste a forças bastante intensas e é responsável pela resistência e rigidez da estrutura devendo ter resistência ao corte de, no mínimo, 1.000 psi. Esse tipo de produto permite a montagem de materiais similares entre si ou dissimilares, e, ao mesmo tempo, participa da integridade estrutural e robustez da peça final (Silva et al., 2007).

O consumo desses adesivos para a junção de metais surgiu na década de 1940, quando os adesivos tornaram-se viáveis comercialmente (Santos, 2005). Sua aplicação está tendo uma evolução significativa, envolvendo montadoras de veículos de transporte de carga e de 
passageiros, indústria aeronáutica, tubulações, peças técnicas em geral, entre outras (Quini, 2011; Bishopp, 2011).

A utilização desses adesivos apresenta algumas vantagens quando comparado a outros métodos de colagem. Essas vantagens estão relacionadas à produtividade, investimentos e estética final do produto, nas quais a aplicação não exige uma mão de obra qualificada para operação e nem equipamentos sofisticados (Fruet, 2011).

Indústrias automotivas dão atenção especial a essa tecnologia devido à redução de peso dos seus produtos. Os adesivos são mais leves que os sistemas mecânicos tradicionais. Como conseqüência, os produtos tornam-se mais leves e, além disso, o processo apresenta menor custo (Fruet, 2011; Mendes, 2005; Feraboli e Masini, 2004).

Normalmente os adesivos estruturais são bicomponetes, ou seja, uma parte é considerada como adesivo e a outra como catalisador. Essa mistura é encontrada em diversas proporções. As bases mais comuns para adesivos são: uretânica, epóxido e acrílicos.

Os adesivos de base uretânica podem ser usados para unir diferentes tipos de materiais sendo adesivos bastantes eficientes e resistentes a uma larga faixa de temperatura. Uma vantagem em relação a outras famílias de adesivos estruturais é sua alta flexibilidade, podendo chegar a até $120 \%$ de alongamento antes da sua ruptura em aplicações estruturais (Ebnesajjad, 2011).

Os adesivos epóxidos apresentam uma grande diversidade de aplicação em virtude da sua alta resistência mecânica e fácil aplicação. Possuem alta resistência à temperatura podendo chegar a até $204^{\circ} \mathrm{C}$. Também são resistentes a solventes, óleos e umidade, porém possuem um baixo alongamento, atingindo em torno de 5\% (Katnam, et al. 2012; Ebnesajjad, 2011).

Os adesivos acrílicos atualmente são os que mais evoluem nas indústrias. Devido a suas características funcionais e uma excelente qualidade de acabamento final, estão tendo resultados significativos de crescimento industrial. Os acrílicos resistem a condições ambientais violentas, possuem alongamento de até $35 \%$ e não necessitam de uma preparação de superfície do substrato rígida (Ebnesajjad, 2011).

Tendo em vista a aplicabilidade de adesivos estruturais nas indústrias automotivas, aeroespaciais e demais ramos correlatos, é importante discutir e analisar qual adesivo estrutural apresenta melhor desempenho na adesão de metais. Realizando-se ensaios normatizados de tração em adesivos estruturais de diferentes bases, pode-se comparar e discutir a força e a falha características do produto, qualificando-o para a indicação pretendida, justificando-se, assim, o desenvolvimento deste trabalho.

O objetivo geral deste trabalho foi comparar o desempenho de adesivos estruturais de diferentes bases na junção de metais, realizando ensaios de resultados de carga em três tipos de adesivos (acrílico, epóxi e poliuretano) na junção de chapas metálicas, através de testes de cisalhamento e tração. A comparação foi feita com corpos de prova expostos ao ambiente natural e à névoa salina, verificando a sua durabilidade, resistência e desempenho em testes de tração. 


\section{MATERIAL E MÉTODOS}

Para a realização do experimento foram utilizados três adesivos estruturais de diferentes bases, acrílica (A), uretânica (U) e epóxi (E), fornecidos pela empresa Lord Industrial. O adesivo A utilizado foi o Lord 406/19, E, o 380/383 e U, o Fusor 2001/2002. Os adesivos, que são considerados bicomponentes (base e endurecedor), foram selecionados por serem indicados para aplicações metal com metal.

As proporções em massa utilizadas (base:endurecedor), de acordo com o boletim técnico de cada mistura, foram de: 1:0,3 para A; 1:0,95 para E e 1:0,86 para U. A leva um tempo de 24 horas para cura, já E e U levam um tempo de 72 horas. Esses parâmetros foram respeitados antes da realização dos ensaios mecânicos.

Os corpos de prova de aço tiveram suas superfícies de aplicação limpas com solvente metil-etil-cetona (MEK), extraindo óleos e graxas. A região que recebeu o adesivo foi demarcada com uma área de $1 \mathrm{in}^{2}$ (pol $^{2}$ ), conforme a norma ASTM D1002 (2010). Houve aplicação em apenas um dos lados do corpo de prova, logo em seguida foi feita a deposição de pérolas de vidro (glass beads) e em continuidade a outra chapa metálica foi depositada. As pérolas foram utilizadas para evitar perda excessiva de adesivo quando a segunda chapa foi colocada sobre a primeira.

Para medir a resistência dos adesivos, foram executados os testes de lap shear (cisalhamento) e cross peel (tração axial), conforme normas ASTM D1002 (2010) e SAE J1553 (2013), respectivamente. Para lap shear, o adesivo foi concentrado no início do substrato e no centro para cross peel, conforme mostra a Figura 1. As amostras em triplicata foram deixadas em ambiente natural e em exposição à atmosfera salina (salt spray) por 500 horas. O ângulo de exposição à direção principal do fluxo foi de 15 a $30^{\circ}$ em relação à vertical.
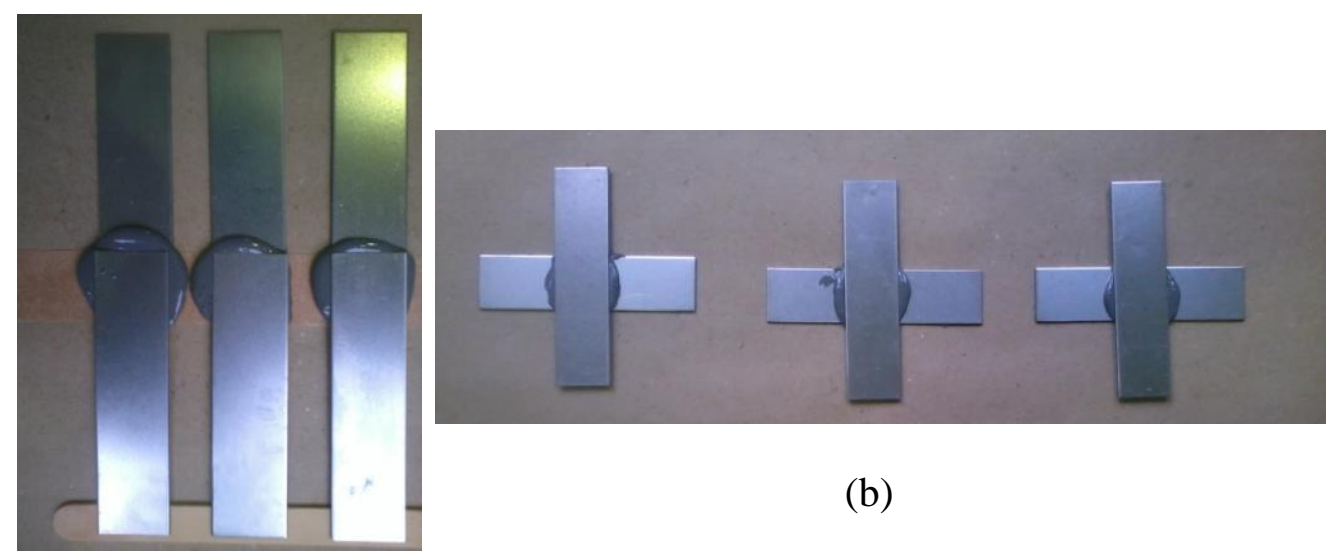

(b)

(a)

Figura 1 - Demonstração das colagens em triplicata para (a) lap shear e (b) croos peel.

Os testes de tração foram efetuados em equipamento EMIC de capacidade de carga de $2.000 \mathrm{~N}$, utilizando-se velocidade de $20 \mathrm{~mm} / \mathrm{s}$. Os dados obtidos foram tratados estatisticamente por análise de variância (ANOVA, fator único, 0,05) na comparação das 
médias e cálculo da diferença mínima significativa pelo teste de Tukey. Em adição, foram feitas as observações qualitativas do tipo de falha ocorrido após a solicitação.

\section{RESULTADOS E DISCUSSÃO}

A Tabela 1 mostra os dados compilados das análises lap shear para o conjunto de colagens expostas ao ambiente natural. Estão indicadas as médias, o desvio padrão e o tipo de falha ocorrido. Na coluna das médias, também são apresentadas letras sobrescritas que indicam se há diferença ou não.

Tabela 1 - Resultados das análises lap shear, para ambiente natural

\begin{tabular}{cccc}
\hline Adesivo & Força média $\left(\mathrm{kgf} / \mathrm{in}^{2}\right)$ & Desvio padrão $\left(\mathrm{kgf} / \mathrm{in}^{2}\right)$ & Tipo de falha \\
\hline $\mathrm{A}$ & $1055,1^{\mathrm{a}}$ & 32,5 & FC \\
$\mathrm{E}$ & $566,4^{\mathrm{b}}$ & 23,8 & FA \\
$\mathrm{U}$ & $976,1^{\mathrm{a}}$ & 31,2 & FA/FC \\
\hline
\end{tabular}

Legenda: Letras diferentes indicam diferença significativa a $5 \% . \mathrm{FA}=$ falha adesiva, $\mathrm{FC}=$ falha coesiva.

Com base nos resultados, nota-se que a força de cisalhamento em A foi maior se comparada à força dos outros dois. Quanto maior a força, melhor é o resultado de desempenho do adesivo. Isso significa que A suporta uma força de cisalhamento maior que os demais adesivos em uma colagem de metal com metal, indicando que tem um possível melhor desempenho em aplicações cujas solicitações mecânicas de cisalhamento sejam elevadas.

O valor da diferença mínima significativa determinada para esse conjunto de resultados foi de 121,9, com valor de q (Tukey) de 4,34. O resultado da força de U ficou mais próximo da de A, o que é notado pelas letras iguais atribuídas às respectivas médias. Por sua vez, E apresentou o menor valor de tração, aproximadamente $44 \%$ mais baixo, e diferente significativamente em relação aos demais. Infere-se que a qualidade de $U$ no tocante à solicitação de cisalhamento não foi considerada inferior à de A. Com base nos dados e no tipo de aplicação, pode ocorrer a escolha de ambos os adesivos. Assim, o fator custo, que não foi analisado neste trabalho, passa a ser preponderante. Em aplicações com tensão cisalhante superior a $570 \mathrm{kgf} / \mathrm{in}^{2}$, a utilização de E deve ser desconsiderada.

Com relação ao tipo de falha observado, A apresentou falha coesiva, $U$ falha mista entre coesiva e adesiva; e E, falha adesiva. A falha coesiva ocorre no interior do próprio adesivo, caracterizada pelo enfraquecimento das forças coesivas internas e permitindo que o adesivo fique retido em ambas as faces do substrato. Em contrapartida, a falha adesiva decorre do desprendimento do adesivo na interface adesivo-aderente, sendo observada que uma das faces fica com a maioria da camada adesiva. Falhas mistas são situações em que não se consegue descriminar corretamente uma falha da outra. O resultado do tipo de falha que o adesivo exprime é tão importante quanto o resultado da força mecânica. A falha de substrato (FS) é o tipo de falha ideal para um excelente resultado, indicando que o adesivo suportou solicitações mecânicas elevadas e permitiu que o substrato fosse deformado. No caso de corpos de prova de aço, devido à alta rigidez, esse tipo de falha dificilmente ocorre. Nessa situação, pode-se considerar FC como o tipo de falha mais aceitável. 
A Tabela 2 mostra os dados compilados das análises cross peel para o conjunto de colagens expostas ao ambiente natural. Estão indicadas as médias, o desvio padrão e o tipo de falha ocorrido. Na coluna das médias, também são apresentadas letras sobrescritas que indicam se há diferença ou não.

Tabela 2 - Resultados das análises cross peel, ambiente natural

\begin{tabular}{cccc}
\hline Adesivo & Força média $\left(\mathrm{kgf} / \mathrm{in}^{2}\right)$ & Desvio padrão $\left(\mathrm{kgf} / \mathrm{in}^{2}\right)$ & Tipo de falha \\
\hline $\mathrm{A}$ & $29,00^{\mathrm{a}}$ & 4,15 & FS \\
$\mathrm{E}$ & $15,36^{\mathrm{b}}$ & 4,78 & FA \\
$\mathrm{U}$ & $30,46^{\mathrm{a}}$ & 0,87 & FS \\
\hline \multicolumn{2}{l}{ Letras diferentes indicam diferença significativa a 5 \%. FA = falha adesiva, FS = falha de substrato. }
\end{tabular}

Com base nos resultados, nota-se que a força de tração axial em $U$ foi maior se comparada à força dos outros dois. Quanto maior a força de tração, melhor é o resultado de desempenho do adesivo. Isso significa que U suporta uma força de tração axial maior que os demais adesivos em uma colagem de metal com metal, indicando que tem um possível melhor desempenho em aplicações cujas solicitações mecânicas de tração axial sejam elevadas.

O valor da diferença mínima significativa determinada para esse conjunto de resultados foi de 9,25, com valor de q (Tukey) de 4,34. O resultado da força de A ficou mais próximo da de $\mathrm{U}$, o que é notado pelas letras iguais atribuídas às respectivas médias. Por sua vez, E apresentou o menor valor de tração, aproximadamente $48 \%$ mais baixo, e diferente significativamente em relação aos demais. Infere-se que a qualidade de A no tocante à solicitação de tração não foi considerada inferior à de U. Com base nos dados e no tipo de aplicação, pode ocorrer a escolha de ambos os adesivos. Assim, o fator custo, que não foi analisado neste trabalho, passa a ser preponderante. Em aplicações com tração superior a 16 kgf/in², a utilização de E deve ser desconsiderada.

Ambos A e U apresentaram a falhas ideais, caracterizadas pela deformação do substrato (FS). E apresentou FA, assim com ocorreu no cisalhamento, indicando um desempenho menos satisfatório.

A Tabela 3 mostra os dados compilados das análises lap shear e a Tabela 4 os de cross peel para o conjunto de colagens expostas à névoa salina. Estão indicadas as médias, o desvio padrão e o tipo de falha ocorrido. Na coluna das médias, também são apresentadas letras sobrescritas que indicam se há diferença ou não.

Tabela 3 - Resultados das análises lap shear, névoa salina

\begin{tabular}{cccc}
\hline Adesivo & Força média $\left(\mathrm{kgf} / \mathrm{in}^{2}\right)$ & Desvio padrão $\left(\mathrm{kgf} / \mathrm{in}^{2}\right)$ & Tipo de falha \\
\hline A & $802,6^{\mathrm{a}}$ & 54,3 & FC \\
E & $529,6^{\mathrm{b}}$ & 48,1 & FA \\
U & $836,2^{\mathrm{a}}$ & 44,6 & FA \\
\hline
\end{tabular}

Letras diferentes indicam diferença significativa a $5 \%$. FC = falha coesiva, FA = falha adesiva. 
Tabela 4 - Resultados das análises cross peel, névoa salina

\begin{tabular}{cccc}
\hline Adesivo & Força média $\left(\mathrm{kgf} / \mathrm{in}^{2}\right)$ & Desvio padrão $\left(\mathrm{kgf} / \mathrm{in}^{2}\right)$ & Tipo de falha \\
\hline $\mathrm{A}$ & $23,75^{\mathrm{a}}$ & 1,20 & FS \\
$\mathrm{E}$ & $16,55^{\mathrm{a}}$ & 3,20 & FA \\
$\mathrm{U}$ & $22,98^{\mathrm{a}}$ & 5,45 & FS \\
\hline \multicolumn{2}{l}{ Letras diferentes indicam diferença significativa a $\%$. FA $=$ falha adesiva, FS = falha de substrato. }
\end{tabular}

Após a análise dos dados obtidos, verifica-se que A foi o que sofreu maior queda no valor da tensão de cisalhamento após ser exposto à névoa salina. No caso da tração axial, U foi o que teve o desempenho mais diminuído. Em relação a E, nota-se que o comportamento após a exposição à névoa teve alterações pequenas, indicando uma maior estabilidade. Em geral ocorreu a manutenção das diferenças de forças dos adesivos após serem expostos à névoa salina, quando comparados no mesmo grupo. As reações de oxidação do corpo de prova após a exposição à nevoa, além de interações química da própria névoa com os adesivos provocaram alterações nas forças dos adesivos e indicam situações prováveis de ocorrer em aplicações expostas a ambiente agressivo.

Em relação ao tipo de falhas em cisalhamento, A apresentou novamente FC, já os demais adesivos apresentaram FA. A indecisão para definir o tipo de falha para $U$ em ambiente natural, caracterizando uma falha mista, não ocorreu após a névoa salina. Assim, U apresentou apenas FA após a névoa salina, indicando uma queda no desempenho qualitativo. Para a situação de tração axial, o comportamento das falhas foi mantido.

\section{CONCLUSÃO}

Este trabalho teve a finalidade de comparar três diferentes adesivos estruturais, acrílico, epóxi e uretânico, na adesão de chapas metálicas. Para isso, os testes realizados foram lap shear (cisalhamento) e cross peel (tração axial) em amostras expostas ao ambiente natural e à nevoa salina, após o tempo de cura de cada adesivo. Com base nos resultados obtidos, concluiu-se que o adesivo acrílico teve desempenho superior ao dos demais, por ter apresentado a maior tensão cisalhante $\left(1055 \mathrm{kgf} / \mathrm{in}^{2}\right) \mathrm{em}$ ambiente natural e a maior tração axial $\left(23,75 \mathrm{kgf} / \mathrm{in}^{2}\right)$ em ambiente severo. Além disso, o acrílico apresentou altos valores de tensão cisalhante e tração axial nos outros testes, cujos maiores valores ficaram com o adesivo uretânico. Os resultados alcançados, porém, não foram considerados diferentes significativamente ao nível de significância de $5 \%$. Não somente, o adesivo acrílico obteve falha coesiva em solicitação cisalhante e falha de substrato em tração axial, indicativas de um bom desempenho.

O adesivo uretânico, apesar dos resultados mecânicos não diferentes significativamente em relação ao acrílico, apresentou falha adesiva no teste de tração axial, sendo, portanto, considerado inferior em desempenho. Por outro lado, o adesivo epóxi teve resultados muito inferiores em todas as determinações quantitativas e qualitativas. Destarte, no conjunto de determinações quantitativas e qualitativas, o adesivo acrílico foi considerado o melhor, seguido do adesivo uretânico e, por fim, o adesivo epóxi. 


\section{REFERÊNCIAS}

ASTM D 907. Standard terminology of adhesives. ASTM International, West Conshohocken, PA, 2012, DOI: 10.1520/D0907-12A, www.astm.org.

ASTM D 1002. Standard test method for apparent shear strength of single-lap-joint adhesively bonded metal specimens by tension loading (metal-to-metal). ASTM International, West Conshohocken, PA, 2010, DOI: 10.1520/D1002-10, www.astm.org.

BISHOOP, J. Adhesives for aerospace structures. In: EBNESAJJAD, S. (Ed). Handbook of adhesives and surface preparation: technology, applications and manufacturing. Oxford: Elsevier, 2011.

COGNARD, J. Some recent progress in adhesion technology and science. C. $R$. Chimie, v. 9, p. 13-24, 2006.

EBNESAJJAD, S. Characteristics of adhesive materials. In: EBNESAJJAD, S. (Ed). Handbook of adhesives and surface preparation: technology, applications and manufacturing. Oxford: Elsevier, 2011.

FERABOLI P.; MASINI, A. Development of carbon/epoxy structural components for a high performance vehicle. Composites: Part B, v. 35, p. 323-330, 2004.

FRUET, T. F. Análise para substituição da solda ponto de uma carroceria de chapas finas de aço carbono e aço galvanizado por adesivo estrutural. 2001. Trabalho de conclusão de estágio II curricular (Engenharia Mecânica), Universidade de Caxias do Sul, Brasil.

KATNAM, K. B.; COMER, A. J.; STANLEY, W. F.; BUGGY, M.; YOUNG, T. M. Investigating tensile behavior of toughened epoxy paste adhesives using circumferentially notched cylindrical bulk specimens. Int. J. Adhes. Adhes., v. 37, p. 310, 2012.

MENDES, C. N. R. P. Contribuição ao desenvolvimento de projeto de carrocerias automotivas utilizando adesivo estrutural para junção de chapas metálicas. 2005. 176 f. Dissertação (Mestrado em Engenharia) - Escola Politécnica, Universidade de São Paulo, São Paulo. 2005.

QUINI, J. G. Adesivos estruturais uretânicos aplicados a combinações de compósitos plásticos e metais. 2011. 141 f. Tese (Doutorado em Tecnologia Nuclear - Materiais) Instituto de Pesquisas Energéticas e Nucleares, Universidade de São Paulo, São Paulo. 2011.

SAE J1553. Cross peel test for automotive-type adhesives for fiber reinforced plastic (Frp) bonding. SAE International, 2013.

SANTOS, A. F. Avaliação do comportamento mecânico de um adesivo estrutural epóxi utilizado na indústria automobilística. 2005. 167 f. Dissertação (Mestrado em Engenharia) - Escola Politécnica, Universidade de São Paulo, São Paulo. 2005.

SILVA, L. F. M.; MAGALHÃES, A. G.; MOURA, M. F. S. F. Juntas adesivas estruturais. Coimbra: Publindústria, 2007.

SKEIST, I. Handbook of adhesives. $3^{\text {a }}$ ed. New York: Chapman \& Hal, 1989. 